\section{Cahiers de Narratologie}

Analyse et théorie narratives

13 | 2006

Nouvelles approches de l'intertextualité

\title{
La Quarantaine de Le Clézio et le vertige intertextuel
}

\section{Madeleine Borgomano}

\section{(2) OpenEdition}

\section{Journals}

Édition électronique

URL : http://journals.openedition.org/narratologie/317

DOI : $10.4000 /$ narratologie.317

ISSN : 1765-307X

Éditeur

LIRCES

Référence électronique

Madeleine Borgomano, «La Quarantaine de Le Clézio et le vertige intertextuel », Cahiers de Narratologie [En ligne], 13 | 2006, mis en ligne le 01 septembre 2006, consulté le 19 avril 2019. URL : http:// journals.openedition.org/narratologie/317 ; DOI : 10.4000/narratologie.317

Ce document a été généré automatiquement le 19 avril 2019

\section{(c) (i) (9)}

Cahiers de Narratologie - Analyse et théorie narratives est mis à disposition selon les termes de la licence Creative Commons Attribution - Pas d'Utilisation Commerciale - Pas de Modification 4.0 International. 


\title{
La Quarantaine de Le Clézio et le vertige intertextuel
}

\author{
Madeleine Borgomano
}

\begin{abstract}
«L'espace littéraire est un espace régi par un vertige essentiel. Chaque livre est l'écho de ceux qui l'anticipèrent ou le présage de ceux qui le répèteront. Chacun, pièce impropre et aléatoire d'un ensemble sans fin, donne sur le précédent et le suivant comme ces enfilades de chambres qui peuplent les cauchemars, rêves d'inatteignable. Aucun qui n'apparaisse perdu entre d'infatigables miroirs. ${ }^{1}$
\end{abstract}

1 Plus largement et plus ouvertement que les autres romans de Le Clézio, La Quarantaine ${ }^{2}$ plonge dans ce « vertige essentiel » que l'on peut nommer aussi « intertextualité ». Même si l'on définit l'intertextualité, à la manière « restrictive » de Genette comme :

«[...] relation de coprésence entre deux ou plusieurs textes, c'est-à-dire, eidétiquement et le plus souvent, par la présence effective d'un texte dans un autre. Sous sa forme la plus explicite et la plus littérale, c'est la pratique traditionnelle de la citation [...] sous une forme moins explicite et moins canonique, celle du plagiat [... ] qui est un emprunt non déclaré mais encore littéral; sous forme encore moins explicite et moins littérale, celle de l'allusion $[. ..] »^{3}$

2 Cette définition restreinte, qui fait de l'intertextualité un canton de « l'hypertextualité » et évite « de verser la totalité de la littérature universelle dans le champ de l'hypertextualité $»^{4}$ atténue un peu le vertige.

3 Mais faut-il l'atténuer quand il s'agit de Le Clézio? Le vertige ne serait-il pas, au contraire, comme un état privilégié, tant pour les personnages que pour l'écriture même ? Ce vertige qui naît « à l'extrémité de la terre » (167), au moment de passer « de l'autre côté », ou encore au bord de la mort (250), cet état instable, ne serait-il pas comme la source même de l'écriture?

4 À la réduire encore à la citation explicite, l'intertextualité dans La Quarantaine reste vertigineuse non seulement par ses dimensions mais par sa diversité et son hétérogénéité.

Robinson sur son île

5 Il est remarquable que le plus évident modèle du roman, qui pourrait être son « hypotexte ", c'est-à-dire « le texte sur lequel il se greffe d'une manière qui n'est pas 
celle du commentaire $»^{5}$, n'apparaisse qu'une seule fois, explicitement, mais comme incidemment :

«Avec sa barbe mal taillée, ses cheveux trop longs collés sur son cou, sa chemise déchirée et ses souliers gris de poussière, il [Jacques] ressemble à Robinson sur son île. » (245)

C'est peut-être qu'il s'agit là d'une relation presque trop évidente, qui n'a pas besoin d'être dite pour s'imposer au lecteur, d'un "architexte», presque d'un genre. La Quarantaine se donne bien, après tant d'autres, comme une variation sur l'histoire de Robinson. Certes, il n'y a pas de naufrage, mais une épidémie, Robinson n'est pas seul, mais les passagers n'en sont pas moins enfermés dans l'île et réduits à la vie sauvage. Entre les deux groupes, les blancs et les " coolies indiens ", passe " une ligne imaginaire " (143) qui sépare "deux mondes" selon les mêmes critères raciaux que ceux qui distinguaient Vendredi de Robinson. Sans devenir par inversion, comme dans le roman de Tournier, l'histoire de Vendredi, La Quarantaine est l'histoire d'un homme qui opte pour «le côté des parias ».

7 Cet intertexte essentiel se double d'un renvoi «autotextuel» de Le Clézio à ses livres précédents : Le Chercheur d'or ${ }^{6}$ et Voyage à Rodrigues ${ }^{7}$. L'île devient l'un des grands lieux le cléziens et greffe l'œuvre sur toute une littérature et toute une mythologie.

Quelques autres textes sont cités ça et là, de façon plus localisée: Bel Ami sert à surnommer l'odieux « Véran de Véreux » (96). Les souvenirs de Splendeurs et misère des courtisanes servent à Léon à évoquer, pour Suryavati, les merveilles des villes, Jacques est comparé à Robinson (245) et Phileas Fogg apparaît dans un rêve de Léon (427).

9 Ces citations qui restent anecdotiques ne sont pourtant pas Insignifiantes. Elles contribuent à souligner clairement, quoique discrètement, «l'architexte » dont relève ce roman,

Du côté de l'Inde

10 Ce n'est pourtant pas la référence à Robinson qui s'affiche à l'orée du roman, mais un intertexte issu d'une tout autre culture. Avant même la première page, le lecteur trouve une épigraphe tirée du Baghavat Purana I, 3, 26 :

«Au crépuscule de cet âge quand tous les rois seront des voleurs, Kalki, le Seigneur de l'Univers, renaitra de la gloire de Vishnou ».

11 Cette prophétie, très obscure, au moins pour un lecteur occidental peu expert en hindouisme, restera inexpliquée dans le roman. Elle s'inscrit donc à son entrée comme une formule mystérieuse, mais pleine d'espoir puisque « le crépuscule » semble compensé par une résurrection. Malgré le défaut d'éclaircissement, cette citation est corrélée à toute une thématique du roman et devient par là signifiante. L'épigraphe, en l'occurrence, pourrait être définie, à la manière de Genette, comme paratexte à statut intertextuel. Car faute d'être élucidée, elle est au moins accompagnée d'une référence. Les Baghavat Purana (Purana = Antiques) sont des livres sacrés des dévots de Vishnu, révéré aux Indes sous le nom de «Bhagavant : le gracieux Seigneur ». Ils sont rédigés en sanscrit et datent $\mathrm{du} \mathrm{X}^{\mathrm{e}}$ siècle. Les Purana racontent les légendes et les mythes des dieux hindous. Vishnu est le Dieu Suprême. Il se manifeste dans des "avataras" («descentes»). Kalki (Kalkin?) serait l'incarnation à venir, qui accompagnera la destruction universelle.

L'épigraphe renvoie donc à l'Inde, à ses religions, à sa mystique. D'abord énigmatique, elle s'éclaire un peu à la lecture du texte, où l'on découvre que toute une partie du roman lui 
fait écho ${ }^{8}$. Dans l'île Plate, où les passagers venus de France pour Maurice sont retenus par l'épidémie « de tout [...] la malaria, la variole, le choléra » (173), la peur et la folie de quelques-uns instaurent une frontière entre le côté de la Quarantaine et l'autre côté, celui des coolies indiens, des parias pour la plupart.

13 Mais, cette frontière, le personnage principal, Léon, qui devient aussi narrateur dans la longue partie centrale du roman, la refuse et la transgresse. Léon «le Disparu », c'est ce grand-oncle dont le narrateur principal porte le prénom et dont il recherche, en vain, les traces. S'il a disparu, ce Léon, c'est justement qu'il est passé de l'autre côté en trahissant son camp, comme le pense son frère Jacques. Ou plutôt qu'il a opté pour ce qu'il avait d'indien en lui, car il est fils d'une eurasienne, et lui-même assez semblable à un coolie et qu'il a épousé le sort de Suryavati, la femme rencontrée sur l'île, elle aussi eurasienne, puisque sa mère, née anglaise, était devenue indienne, de la plus basse caste, celle des Doms chargés de brûler les morts et de veiller sur les bûchers.

14 Ce choix renverse le système de valeurs dans lequel baignent les autres passagers européens. Il instaure les valeurs opposées du renoncement et du dénuement. On y reconnait les choix permanents de Le Clézio magnifiant le désert, ou la culture des indiens d'Amérique.

Dans La Quarantaine, ces valeurs appartiennent à l'Inde, celle au moins des mystiques. Celle aussi, par nécessité, des parias et des pauvres. Ainsi, la citation en épigraphe remplit - elle bien ce que Genette désigne comme la deuxième fonction de ce texte en exergue, celle de "commentaire du texte, dont elle précise ou souligne la signification ${ }^{9}$. Elle annonce aussi et ouvre, littéralement et sémantiquement, un large champ intertextuel indien.

La chanson du voleur

À la prédiction sibylline : "Quand les rois seront des voleurs ", qui pourrait s'interpréter (littéralement) comme l'attente ou l'annonce d'un monde renversé, fait écho la chanson de Surya, héritage de sa grand-mère. Giribala l'a entendue pour la première fois sur le radeau qui descendait la rivière Yamuna, chantée comme une berceuse pour un enfant mourant, dans une langue étrange :

«[...] il lui a semblé que chaque parole entrait en elle pour toujours, comme si elle était chargée d'un sens mystérieux :

«Chhurm, kala, chalo gul layé, voleur, ô voleur, viens, entrons dans cette demeure, enlève tes chakkal, prends tout, bhimté, bagelé, allume le ghasai, et toi, litara, jette la boule de terre, le neola, si tu entends un bruit! Kajjachamaa, un espion te guette! Thipja ! Cache toi ! Palwé hoja! Gare à toi ! Kainkar kar ! Jette une motte de terre ! Lalli lug gaya, Kala lug gayé, le vol est fini et le voleur est mort !» $(201,202)$

17 Le texte n'éclaircit guère le "mystère » de la chanson. À peine est-il précisé que ce « langage doux et glissant » est « la langue secrète des Doms », qui sont, selon Surya, « des vagabonds et des voleurs » (407).

Le jeu intertextuel instaure, dans le texte, une zone d'ombre où se dissimule le mystère, l'étrangeté de "l'autre côté ». Peut-être même, ce vol, cette effraction finissent - ils par devenir comme une figure masquée et inquiétante du passage qu'accomplit Léon vers un monde qui n'est pas le sien. Un passage dont on ne sort pas indemme : « Le vol est fini et le voleur est mort ».

Le motif du voleur s'enrichit encore d'un renvoi autotextuel. Le lecteur fidèle de Le Clézio se souvient du titre d'une nouvelle : «Ô voleur, voleur, quelle vie est la tienne? » qui figure dans le recueil La ronde et autres faits divers ${ }^{10}$, et raconte l'histoire navrante d'un 
immigré portugais devenu voleur. Ce titre était lui-même la traduction d'un vers d'une chanson traditionnelle portugaise, dont le texte original fermait la nouvelle $(R, 234,235)$.

Cette épigraphe énigmatique débouche sur un champ intertextuel très riche et très ouvert. Il déclenche - mais seulement chez un Lecteur Modèle ! - une véritable surcharge interprétative.

Les textes sacrés hindous

L'épigraphe ouvre un autre intertexte, celui des légendes sacrées de l'Inde, rassemblées autour de la Yamuna. La Yamuna c'est d'abord une longue rivière de l'Inde du Nord, (1370 $\mathrm{km}$ ) affluent du Gange qui arrose la ville de Delhi. «La Yamuna » est le titre du récit enchâssé, qui alterne, dans la partie centrale du roman, avec le récit du «Disparu » racontant la Quarantaine. Dans "La Yamuna », le narrateur premier, le second Léon, reprend la parole pour « inventer» l'histoire de Giribala, fuyant, avec Ananta, l'enfant qu'elle a recueillie, la guerre et les massacres, conséquences de "la révolte des Cipayes » (nom francisé des soldats indigènes recrutés dans l'armée des Indes par les Anglais et nommés dans le roman «sepoys ») qui ensanglanta l'Inde du Nord en 1857-1858. «La Yamuna » raconte une lente descente du fleuve jusqu'à Calcutta.

Mais la Yamuna est aussi l'un des sept fleuves sacrés de l'Inde. C'est sur ses bords que serait né le Seigneur Krishna, l'un des « avatara » de Vishnou, dont les Purana racontent l'enfance bucolique (501). Giribala baptise sa fille adoptive dans les eaux sacrées de la Yamuna et lui donne le nom "dicté par le fleuve, «Ananta ", l'Éternel, le serpent sur lequel Dieu se repose jusqu'à la fin du monde » (199).

Surya raconte encore une autre légende, celle de Yama, dieu des morts :

"Yama est fils du soleil, il attend sa sœur, la rivière Yamuna. Quand elle vient, elle allume un grand feu et avec la cendre, elle marque le front de son frère, comme j'ai fait, pour que leur amour ne finisse jamais. » (225)

Ainsi, celle que le narrateur nomme «Suryavati », «force du soleil » ( 87) «à cause de la reine du Cachemire [...] dans le livre de Somadeva ${ }^{11} »(87)$ sert de médiatrice avec la culture indienne. Elle-même n'a jamais connu l'Inde: elle a reçu cette connaissance oralement, par les récits de sa mère, qui elle-même les tenait de sa propre mère, Giribala. L'Inde n'entre donc dans le roman que de façon très médiatisée, comme une légende transmise par les femmes. Cette forme mythique d'existence reste par définition assez floue. Mais elle exerce un attrait d'autant plus puissant sur le narrateur qu'il est amoureux de son interprète et que tous les récits deviennent signes d'amour.

Une allusion très brève est faite à une autre légende hindoue, celle d'Angoli Mala :

"Suryavati m'a dit que je ressemblais à Angoli Mala, le bandit qui coupait les doigts

des gens dans la forêt et que Bouddha a guéri de sa folie. » (383)

Le bref récit ouvre sur une auto-intertextualité perceptible a posteriori seulement, puisque l'avant-dernier livre de Le Clézio, paru en 1999, contient une nouvelle intitulée « Angoli Mala » qui transpose l'histoire du bandit dans l'univers des indiens d'Amérique du Sud et fait de lui un rebelle, comme Léon le Disparu, mais sur un mode plus violent. L'intertexte tisse des liens qui ne se soucient pas du temps ni de l'espace.

27 Finalement, la fascination pour une culture indienne largement fantasmée et impossible à rejoindre, comme il est impossible de retrouver les traces du Disparu - débouche sur une philosophie de l'illusion:

«Alors tout est inventé, illusoire, comme la vie qui continue autrement quand on poursuit un rêve, nuit après nuit. » (531) 
Rimbaud

Un deuxième intertexte radicalement autre (au moins en apparence) se manifeste dès l'incipit du roman, implicitement par le titre, "Le voyageur sans fin ", et explicitement, après un léger suspense, dès le second paragraphe. Le personnage dégingandé qui « apparaît [...] dans l'encadrement [de la porte], contre la nuit » (15) est alors nommé et situé dans le temps historique :

« Je pense à la façon dont mon grand-père a vu Rimbaud, la première fois. C'était au début de l'année 1872 [...]» (15) oralement, mais d'abord par les hommes. Léon (le Disparu) a entendu l'histoire de l'apparition du jeune Rimbaud à la porte d'un café enfumé, dans le froid «mortel » de l'hiver parisien, de la bouche de son frère Jacques. Il a vécu lui-même la seconde rencontre avec l'homme malade, presque agonisant, dans un hospice d'Aden, en 1891, l'année même de la mort du poète. Le chapitre s'intitule "L'empoisonneur ", car Rimbaud, hanté par les chiens du Harrar, leur jetait des morceaux de viande empoisonnée. L'autre Léon, le descendant, a entendu ces récits de sa grand-mère Suzanne (19). Là aussi l'amour pour la conteuse ( «'ai beaucoup aimé ma grand-mère Suzanne ») et la solennité de la transmission alimentent la légende :

«Fais bien attention. Ce que je vais te dire est authentique, je n'ai rien ajouté. Quand tu auras des enfants, il faudra que tu le leur racontes exactement comme je te l'ai dit. » (19) Disparu, autour du rêve de départ, de la rébellion contre l'ordre :

«Je n'ai pas trouvé celui que je cherchais. Peut-être que, comme Rimbaud à qui j'ai voulu qu'il ressemblât, sa vie est devenue sa légende. » (530)

«Je » désigne ici le narrateur premier, le petit-fils. En même temps se tissent de nombreux échos. Ainsi, dans la dernière partie du roman, Anna, la survivante, porte elle aussi aux chiens errants des boulettes empoisonnées, refaisant le geste de pitié et de terreur du poète mourant.

Rimbaud est présent aussi comme poète : «Léon avait appris par cœur ses poèmes, Le bateau ivre, Voyelles, Les assis, que Jacques avait recopiés pour lui dans ses cahiers d'écolier » (25). La poésie du « voyou » (29) est transmise, avant que ne soient " défaits tous les liens [familiaux]» (17) par le frère encore bien-aimé, qui pourtant prendra le parti de l'ordre. Et, finalement, comme l'homme du Harrar, Léon lui-même reniera la poésie :

«Il n'y a plus de poésie. Je n'ai plus envie de lire les longues phrases un peu solennelles de Longfellow. Il me semble que même les mots violents de l'homme d'Aden ont disparu dans le ciel, ils ont été emportés par le vent et perdus dans la mer. » (409)

Mais, plus essentielle encore que toutes ces correspondances, se profile, à l'arrière-plan, une allusion tacite à la formule fameuse de Rimbaud, dans « La lettre du voyant » : « Je est un autre ». La phrase n'est jamais citée, mais plusieurs fois paraphrasée. Ainsi, juste après avoir raconté la première rencontre de son grand-père Jacques avec Rimbaud, le narrateur premier, le petit-fils, note : 
«Parfois il me semble que c'est moi qui ai vécu cela. Ou bien que je suis l'autre Léon, celui qui a disparu pour toujours, et que Jacques m'a tout raconté quand j'étais enfant. » (21)

On remarque que «l'autre » est en italiques, justement. Un peu plus loin « il me semble » s'est effacé pour laisser place à une affirmation: «Je suis devenu Léon Archambau, le Disparu » (26). Même si le doute revient finalement :

"Je l'ai regardé si souvent [cette photo] que parfois il me semblait que j'oubliais qui

j'étais, comme si j'avais changé de corps et de visage. Alors j'étais Léon, l'autre Léon

$[\ldots] »(531)$

Le dédoublement se complique de ce que, au-delà de ces deux Léon, il y a l'écrivain Le Clézio, qui n'est pas étranger à toute cette histoire. Le texte, à travers les vertiges de son énonciation, démultiplie l'identité même, « perdue entre d'infatigables miroirs ».

Et peut-être même pourrait-on faire de «Je est un autre » la formule même de l'écriture romanesque, quitte à choquer philosophes et poètes.

D'autres poètes

À ces deux orientations intertextuelles déjà fortement divergentes au niveau culturel, spatial et temporel, s'ajoutent de très nombreuses autres références. Dès les premières pages, le lecteur rencontre une énumération de noms de poètes, ceux qu'aimait Suzanne, la grand-mère du narrateur : «Shelley, Longfellow, Hugo, Hérédia, Verlaine » (20). Sont également cités plusieurs poèmes de Baudelaire, le plus souvent en relation de correspondance thématique avec le roman. Même L'ennemi (31), qui peut sembler hétérogène, souligne en fait le jeu mélancolique avec le temps qui construit ce texte :

"Ô douleur ! ô douleur ! le temps mange la vie »

La mer (32), Parfum exotique (294), L'invitation au voyage, (295) développent des harmoniques plus évidentes avec l'argument principal.

Le poète américain Longfellow ${ }^{12}$ occupe une place privilégiée. Le recueil de ses poésies, un petit livre bleu et noir (308), est l'unique livre que les prisonniers de l'île Plate ont avec eux pendant la quarantaine (64). Longtemps, il devient aussi leur seul réconfort.

De longs extraits des poèmes de Longfellow sont cités, en anglais dans le texte. Le poème préféré de Suzanne, Fata Morgana (20), Birds of Passage (31) ou La cité de la mer :

« The panting City cried to the Sea

I am faint with heat - Oh breathe on me !

$[\ldots]$

Silent as dreams are, and sudden as sleep

Life-giving, death giving, which will it be,

O breath of the merciful, merciless sea?» (370)

Suzanne et Léon lisent et relisent aussi The song of Hiawatha $(295,308,409)$.

Ce poème reprend les légendes des indiens d'Amérique, autour d'un demi-dieu envoyé par le Grand-Esprit pour enseigner aux hommes les arts de la paix. Mais le personnage, édulcoré et christianisé, «appartient à ces images conventionnelles si nombreuses qui permettent à l'Américain [...] de tolérer ces groupes encore non assimilés dont l'existence réelle [...] troublerait la paix de sa conscience. $»^{13}$

Les nombreuses références à un texte dont la philosophie assimilationiste est en contradiction radicale avec celle de respect de l'altérité qui ressort du livre de Le Clézio, peuvent surprendre. Mais on remarquera qu'aucune allusion n'est faite ni à l'identité de Hiawatha, ni au contenu narratif du chant. Les vers cités sont descriptifs et parlent de 
mer et de soleil. L'usage qui est fait du poème, comme "récompense » et comme consolation est même nettement explicité :

«Je lis The Song of Hiawatha comme si c'était un conte pour enfants, sans signification cachée, simplement une musique de mots, pour faire rêver. Parfois il me semble que je lis interminablement le même passage.

"Can it be the sun descending

O'er the level plain of water ? [...]» (308)

À l'instar de Baudelaire, et des autres poètes cités, même si l'on peut découvrir quelques correspondances thématiques (exotisme, évasion etc..), c'est pour la «musique» et le « rêve » que ces poèmes sont présents dans le roman.

En même temps, cette interférence permanente avec la poésie est en accord profond avec l'une des qualités majeures de l'écriture de Le Clézio et en particulier dans ce roman :

"J.M.G. Le Clézio est notre seul grand romancier depuis longtemps, à vouloir faire marcher d'un même pas la fiction en prose et la plus haute poésie. [...] Pas d'ornement ici et pas de contours flous, pas de tarabiscot ni d'effets de voix. La poésie ne vient pas au secours du roman pour en maquiller les imprécisions et les faiblesses ; elle naît de la prose elle-même, de sa rigoureuse netteté, de la précision des descriptions, de la générosité du regard. ${ }^{14}$

Le Clézio écrit, en effet, des « romans " peu narratifs et de peu de matière, qui demandent à être lus comme des poèmes. C'est peut-être cette invitation à un mode de lecture que suggère la présence dans le texte, de tant de poèmes.

Les carnets du botaniste

Dans la partie centrale du roman, qui porte le titre redondant de "La Quarantaine", Léon, narrateur principal, cède régulièrement la parole au «Journal du botaniste » (69), John Metcalf. Les pages du journal, quoique tout aussi fictives que le reste, sont présentées comme des citations, en italiques, et d'ailleurs leur contenu informatif authentique est bien extrait d'un traité de botanique. On ne peut donc parler, rigoureusement, d'intertextualité, puisque les deux textes ont le même statut. Pourtant, il y a bien là importation, localisée, non d'un texte, mais d'un langage spécialisé. Car ce journal est celui du botaniste, exclusivement. Presque aucune allusion n'est jamais faite aux événements, pourtant tragiques, que vit l'homme. À peine une phrase de temps en temps : «La fièvre et une mauvaise nuit m'ont tenu couché toute la journée d'hier » (95). Ou encore : « L'après-midi, malgré la fatigue, retourné à la pente est du volcan. » (153)

En pur scientifique, le botaniste se contente de repérer, répertorier, situer et nommer les plantes. Il aligne des phrases sèches, souvent nominales, sans commentaires, sauf des remarques sur les usages possibles de ces plantes, car il cherche une légumineuse qui pourrait pallier la disette. Il est, en effet, animé d'une forte conviction que Léon finit par partager :

«Ce sont les plantes qui sauvent les hommes » (268)

Cependant les citations du journal s'espacent peu à peu. La dernière, très courte, n'est plus précédée que d'une date incomplète « 21 juin?» (203). Elle est aussi suivie de ces mots de Léon : « Ils ont emmené John Metcalf ce matin ». Emmené à l'îlot Gabriel, où l'on isole et relègue les mourants. Alors, la sécheresse et la sobriété du journal, l'énumération de tous ces noms savants, et l'effacement de l'observateur deviennent bouleversants.

51 Ainsi, l'écriture de Le Clézio se réclame-t-elle, en somme, de deux modèles apparemment contradictoires : la poésie lyrique et ses grands rythmes musicaux, et la transparence 
d'un texte descriptif et sobre. C'est dans cette contradiction, jamais résolue, qu'elle trouve son dynamisme.

Le jeu d'une intertextualité abondante et diverse relève du même dynamisme contradictoire. Le Clézio ne prétend pas à l'invention ni à l'originalité. Il dit à Gérard Cortanze :

«[...] je pense qu'en fait, lorsqu'on écrit, on n'invente pas. On est toujours propulsé par une mémoire qui appartient quelquefois aux autres $[. ..] »^{15}$

Ou plutôt, il reconnait ses dettes. Marguerite Duras disait: «J'écris avec. ${ }^{16}$ Le Clézio aussi « écrit avec »; avec les romans d'aventure de sa jeunesse, avec ses poètes préférés, avec les grands textes mythiques du monde mais aussi avec les notations austères des scientifiques. Il n'imite pas, il ne plagie pas : il fait son miel et construit son propre espace sans tenir compte des frontières géographiques, culturelles ou textuelles. Il y a bien là un « vertige essentiel » et d'ailleurs Le Clézio aime le «vertige » qui saisit ses personnages devant le ciel, la mer, la ligne des montagnes trop longtemps contemplées (412), ou sous l'effet du soleil trop fort (270).

\section{NOTES}

1. Michel Schneider, Voleurs de mots, Paris, Gallimard, 1985, p. 81.

2. Jean-Marie Gustave Le Clézio, La Quarantaine, Paris, Gallimard, 1995, ici Folio.

Abréviation : Q. Les chiffres entre parenthèses renvoient tous aux pages de La Quarantaine, ed. Folio.

3. Gérard Genette, Palimpseste, Paris, Seuil, 1982, p. 8.

4. Ibid., p. 16.

5. Ibid., p. 11.

6. Le Clézio, Le Chercheur d'or, Paris, Gallimard, 1985.

7. Le Clézio, Voyage à Rodrigues, Paris, Gallimard, 1986.

8. Encyclopædia Universalis, article « Vishnu » et « Purana ».

9. Gérard Genette, Seuils, Paris, Seuil, 1987, p. 146.

10. Le Clézio, La ronde et autres fais divers, Paris, Gallimard, 1982 (Folio). Abréviation, $R$.

11. Somadeva ( $\mathrm{XI}^{\mathrm{e}}$ siècle) est l'auteur d'un très volumineux recueil de contes (enchâssés dans un récit-cadre) écrit en sanscrit et très célèbre aux Indes.

12. Henry Wadsworth Longfellow, 1807, 1882.

13. Dictionnaire des personnages, article « Hiawatha », Paris, Robert Laffont, col. Bouquins 1960.

14. Pierre Lepape, « Le radeau ivre », in Le Monde des livres, 20 octobre 1995.

15. Gérard de Cortanze, Le Clézio, vérité et légendes, Paris, Éditions du Chêne, 1999, p. 51.

16. Marguerite Duras à Montréal, Montréal (Québec), Spirale, 1981, p. 23. 


\section{RÉSUMÉS}

Le terme de vertige employé par M. Schneider convient particulièrement pour qualifier les jeux de l'intertextualité dans La Quarantaine de Le Clézio, même en la limitant aux textes effectivement cités. Intertexte essentiel, et trop évident, Robinson n'est nommé qu'une fois. L'épigraphe, la chanson du voleur et les références aux textes sacrés hindous rattachent le roman à l'Inde et à ses valeurs, qui constituent l'un des pôles signifiants, le côté des parias que Léon (le Disparu), narrateur second, épousera en même temps que Suryavati. Un autre aspect de l'intertexte se rattache aux deux figures de Rimbaud, le jeune voyou de Paris et l'homme malade d'Aden. Le renvoi implicite à la formule "Je est un autre» accentue les complexités de l'énonciation et les glissements de l'identité. Les très nombreuses citations de poèmes soulignent la tendance poétique plus que narrative de l'écriture de Le Clézio. Tandis que les citations du « journal d'un botaniste » révèlent la nostalgie d'un discours descriptif savant et neutre. Ainsi, le vertige intertextuel manifeste et multiplie les contradictions dynamiques qui animent l'écriture de Le Clézio et contribuent à la production du sens.

\section{AUTEUR}

\section{MADELEINE BORGOMANO}

Université de Provence Aix-Marseille I 